\title{
Feasibility of intraoperative water testing in aortic valve repair: Direct visualization from left ventricle with a videoscope
}

Shunsuke Miyahara, MD, PhD, ${ }^{\mathrm{a}}$ Takanori Oka, MD, ${ }^{\mathrm{b}}$ Hiroaki Takahashi, MD, PhD, ${ }^{\mathrm{a}}$ Takeshi Inoue, MD, ${ }^{\mathrm{a}}$ Masamichi Matsumori, MD, $\mathrm{PhD},{ }^{\mathrm{a}}$ Hiroshi Tanaka, $\mathrm{MD}, \mathrm{PhD},{ }^{\mathrm{a}}$ and Yutaka Okita, $\mathrm{MD}, \mathrm{PhD}^{\mathrm{a}}$

\begin{abstract}
Objective: We describe a simple method to assess the aortic valve using a videoscope inserted in the left ventricle (LV-VS) during valve-sparing root replacement. The aim of this study was to evaluate the feasibility of this technique by comparing it with the findings of postoperative transesophageal echocardiography (TEE).

Methods: Thirty-six patients (29 male, mean age $45.4 \pm 20.1$ years) undergoing aortic root reimplantation were assessed intraoperatively with LV-VS. The LV-VS was inserted from the right upper pulmonary vein into the left ventricle and set toward the aortic valve. After completion of graft implantation, inspection was performed with LV-VS by pressurizing the neo-sinus before attachment of coronary arteries. Valve competency evaluated by LV-VS was compared with postoperative TEE findings, according to the group of cusp morphologies. Group 1 included 26 patients with tricuspid aortic valve, and group 2 included 9 patients with bicuspid aortic valve and 1 quadricuspid aortic valve.

Results: The grade of aortic regurgitation (AR) improved from $2.9 \pm 1.6$ preoperatively to $0.33 \pm 0.6$ postoperatively $(P<.001$ vs preoperatively). In 4 patients, LV-VS was used only before repair. In group 1, intraoperative LV-VS showed a competent valve in 20 and an incompetent valve in 3 patients, and postoperative TEE showed non/trivial AR in 15, mild AR in 5, and mild-to-moderate AR in 3 patients. In group 2, 9 patients achieved a competent valve on intraoperative LV-VS and non/trivial AR on postoperative TEE.
\end{abstract}

Conclusions: Intraoperative direct inspection with LV-VS is a feasible method for confirming the completion of cusp repair. ( $\mathrm{J}$ Thorac Cardiovasc Surg 2017;154:24-9)

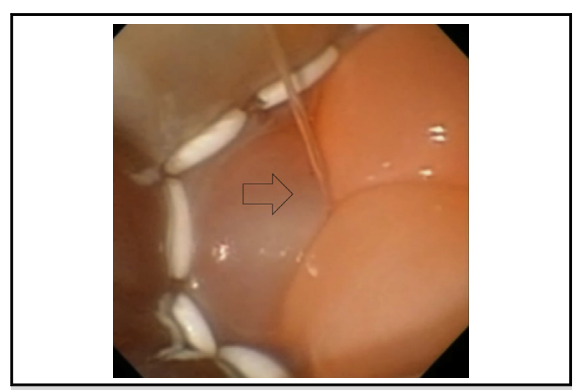

Videoscope showed a competent valve with narrow central stream (open arrow).

\section{Central Message}

Direct inspection with videoscope during valvesparing root replacement is a feasible method to confirm cusp competition and provide enough information for intraoperative decision-making.

\section{Perspective}

The method of intraoperative cusp assessment in aortic valve repair is not as standardized as that of mitral valve repair. Our videoscope system provides information from below the aortic valve, just like water testing in mitral valve repair. This simple method could confirm the completion of cusp repair and provide the surgeon enough information to make an intraoperative decision promptly.

See Editorial Commentary page 30 .

See Editorial page 22.
Although attempts to correct aortic regurgitation (AR) by cusp repair have been made since the beginning of cardiac

From the ${ }^{\mathrm{a}}$ Division of Cardiovascular Surgery, Department of Surgery, Kobe University Graduate School of Medicine, Kobe; and ${ }^{\mathrm{b}}$ Division of Cardiovascular Surgery, Takatsuki General Hospital, Takatsuki, Japan.

Received for publication Oct 17, 2016; revisions received Nov 20, 2016; accepted for publication Dec 28, 2016; available ahead of print March 6, 2017.

Address for reprints: Yutaka Okita, MD, PhD, Kobe University School of Medicine, 7-5-1, Kusunoki-Cho, Chuo-Ku Kobe 650-0017, Japan (E-mail: yokita@med. kobe-u.ac.jp).

$0022-5223 / \$ 36.00$

Copyright (c) 2017 by The American Association for Thoracic Surgery

http://dx.doi.org/10.1016/j.jtcvs.2016.12.061 surgery, ${ }^{1}$ methods for aortic valve repair are not as standardized as those of mitral valve. ${ }^{2}$ During the past 20 years, however, we have achieved steady and significant progress in the field of aortic valve repair accompanied by

Scanning this QR code will take you to a supplemental video for the article. 

Abbreviations and Acronyms
$\mathrm{AR}=$ aortic regurgitation
$\mathrm{CP}=$ cardioplegic
$\mathrm{LV} \quad=$ left ventricle
LV-VS $=$ left ventricular videoscope
TEE $=$ transesophageal echocardiography
VSRR $=$ valve-sparing aortic root replacement

valve-sparing aortic root replacement (VSRR). The indications for VSRR also have been expanded from simple root dilation to complex pathologies of the aortic cusp, which need additional cusp repair. ${ }^{3-5}$ The probability of success of these operations largely depends on the surgeon's experience, which includes making a proper decision intraoperatively.

The assessment of repair quality during VSRR, however, is not established and largely depends on visual inspection from the aortotomy and transesophageal echocardiography (TEE) after coronary resuscitation. The lack of information with adequate imaging of the aortic valve before TEE sometimes limits the application of additional procedures or encourages unnecessary ones. Because of the anatomical restriction, the inspector cannot approach to the aortic valve from the blood inflow side, which means that surgeons cannot directly watch the regurgitation of the aortic valve as we can do with saline testing during mitral valve surgery. We previously reported a case of a novel method to access the AR intraoperatively from left ventricle (LV) with a videoscope (ie, left ventricular videoscope, or LV-VS). ${ }^{6}$ We herein demonstrate the applicability and reproducibility of the LV-VS in different forms of complex aortic cusp pathology and its feasibility in valve-sparing operations.

\section{MATERIALS AND METHODS \\ Operative Technique and Approach to Intraoperative Assessment}

Between October 1999 and March 2016, 250 patients underwent VSRR in our group. We exclusively used the reimplantation technique for VSRR. We developed an intraoperative aortic valve assessment system using LVVS in January $2012 .^{6}$ Thirty-six of 250 patients whose cusp morphology and competency were accessed intraoperatively with LV-VS were enrolled in the study. Details of the operative techniques are described in our previous report. ${ }^{7,8}$

After the replacement of the aortic root with a Valsalva graft (Terumo Medical, Somerset, NJ) with 12 reinforced 3-0 polyester mattress sutures in the first row and continuous 5-0 polypropylene sutures in the second row, cusp repair was performed in cases of necessity. The cusp competency was assessed by the administration of cardioplegic (CP) solution before coronary implantation. The distal edge of the Valsalva graft was clamped beside the tip of the $\mathrm{CP}$ cannula and the neo-sinus was pressurized with $\mathrm{CP}$ solution over $250 \mathrm{~mm} \mathrm{Hg}$. If the pressure of neo-sinus did not increase sufficiently, the leakage of CP solution below aortic valve was suspected, indicating residual AR. Simultaneously, the aortic valve was visualized directly from the LV with an LV-VS (Olympus, Tokyo, Japan), which has a diameter of $8.5 \mathrm{Fr}$ in its tip and $9.9 \mathrm{Fr}$ in inserting portion with the length of $670 \mathrm{~mm}$ and can curve flexibly to UP/DOWN with $180^{\circ} / 275^{\circ}$, respectively. The approach of the scope is right upper pulmonary vein and is inserted parallel to the $L V$ venting tube in a different hole there.

Figure 1 illustrates the insertion of the LV-VS. The left ventricular venting is continued to achieve a clear view. The tip of the scope is set toward the aortic cusp. ${ }^{6}$ The aortic root is again pressurized to observe the competency of the cusp. If the cusp prolapses or residual regurgitation exists, we can detect them directly with LV-VS and add another repair procedure appropriately. The intraoperative findings of LV-VS were classified into following 2 categories. A "competent valve" was defined as follows: there was no leakage of the CP solution from the aortic valve or was only a narrow stream of leakage of the $\mathrm{CP}$ solution with no eccentricity under the condition in which the pressure of the CP circuit increases promptly over $200 \mathrm{~mm} \mathrm{Hg}$. These residual leakages were considered as nonsignificant leakage. Otherwise, an "incompetent valve" included a condition with significant leakage, which was defined as any amount of leakage with eccentricity or massive leakage with wide width of current, which led us to suspect inadequate coaptation of the free margin.

After confirmation of the competency of the aortic cusp, implantation of the coronary arteries and distal anastomoses was completed. Finally, the presence and degree of residual AR were determined with intraoperative TEE, after the patient was weaned off the cardiopulmonary bypass. The observations of valve competency with LV-VS were compared with those of TEE to evaluate the feasibility of LV-VS. The study protocol was reviewed and approved by the institutional review board, and additional informed consent was not required.

\section{Patient Demographics and Echocardiography}

Twenty-nine men and 7 women aged $45.4 \pm 20.1$ years undergoing elective surgery were included in this study. Integrating qualitative and semiquantitative assessment, aortic valve insufficiency was graded as none/trivial: 0 , mild: 1 , mild to moderate: 2 , moderate to severe: 3 or severe: 4 . The mean grade of AR in this patient group was $2.9 \pm 1.6 \mathrm{~Pa}-$ tients were divided into 2 groups according to the cusp morphologies. Group 1 included 26 patients with tricuspid aortic valve and group 2 included 10 patients with cusp anomaly: 9 patients with bicuspid aortic valve and 1 with quadricuspid aortic valve. The majority of the patients

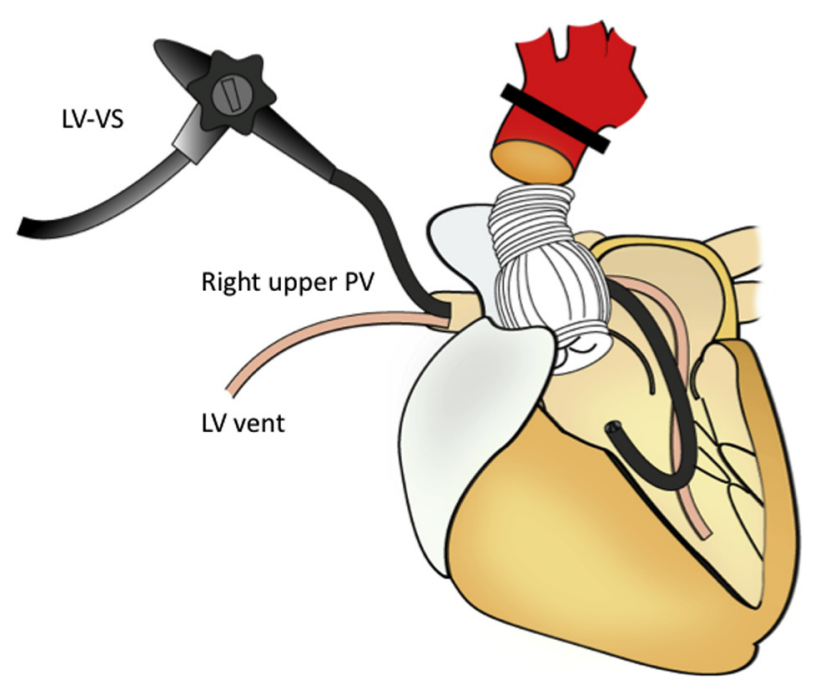

FIGURE 1. Illustration for the insertion of the LV-VS. $L V$-VS, Left ventricular videoscope. 
TABLE 1. Pre- and postoperative echocardiographic parameters

\begin{tabular}{lccr}
\hline \multicolumn{1}{c}{ Variable } & Preoperatively & Postoperatively & $\boldsymbol{P}$ value \\
\hline AR, grade & $2.9 \pm 1.6$ & $0.33 \pm 0.6$ & $<.0001$ \\
LVDd, mm & $55.9 \pm 12.3$ & $44.5 \pm 8.2$ & $<.0001$ \\
LVEF, \% & $58.5 \pm 10.2$ & $54.0 \pm 11.0$ & .0022 \\
AVJ diameter, mm & $25.6 \pm 3.1$ & $23.1 \pm 2.6$ & $<.0001$ \\
Valsalva sinus diameter, $\mathrm{mm}$ & $45.8 \pm 8.8$ & $30.4 \pm 2.9$ & $<.0001$ \\
\hline
\end{tabular}

$A R$, Aortic regurgitation; $L V D d$, left ventricular diastolic diameter; $L V E F$, left ventricular ejection fraction, $A V J$, aortoventricular junction.

presented preoperatively with moderate or greater AR $(n=26,72.2 \%)$; $17(47.2 \%)$ patients had regurgitant pathology of cusp prolapse that was defined by preoperative TEE. According to the recommendations of the American Society of Echocardiography, the AR jet was described as eccentric when the main axis of the jet formed an angle $>45^{\circ}$ with the left ventricular outflow tract or central when the main axis of the jet paralleled that of the left ventricular outflow tract and was directed toward the apex. ${ }^{9}$

\section{RESULTS}

The grade of AR improved from $2.9 \pm 1.6$ preoperatively to $0.33 \pm 0.6$ postoperatively $(P<.0001)$. The aortic root and left ventricular dimension also were reduced significantly (Table 1). The flowchart in Figure 2 describes the intraoperative management that accompanied LV-VS. Postprocedural LV-VS assessment was not performed always, ie, when the cusp pathology was simply repairable and/or we needed to perform another concomitant valve surgery or aortic arch repair. In 4 patients, postprocedural LV-VS assessment was not performed. One of these 4 patients, however, had significant AR with residual cusp prolapse in TEE and a more serious problem in the mitral valve, which was concomitantly repaired. Therefore, a revision both on the mitral and aortic valve was needed after cardiac resuscitation, and finally, both $\mathrm{AR}$ and mitral regurgitation was controlled to an acceptable degree under TEE visualization; an additional plication stitch in prolapsed right coronary cusp and another size of mitral annuloplasty ring was reapplied, respectively. Final LV-VS and postoperative TEE results according to the groups are summarized in Table 2. In both groups, there was no discrepancy between the results of LV-VS and TEE in terms of the presence of significant AR.

In group 1, intraoperative LV-VS showed a finally competent valve in 20 and incompetent valve in 3 . On postoperative TEE, none/trivial AR was observed in 15, mild AR in 5, and mild to moderate AR in 3. Figure 3, A, shows a preprocedural LV-VS with an eccentric jet due to right coronary cusp prolapse coexisting with another central jet, which indicates an incompetent valve with significant AR with eccentricity. In Figure 3, $B$, both the central and

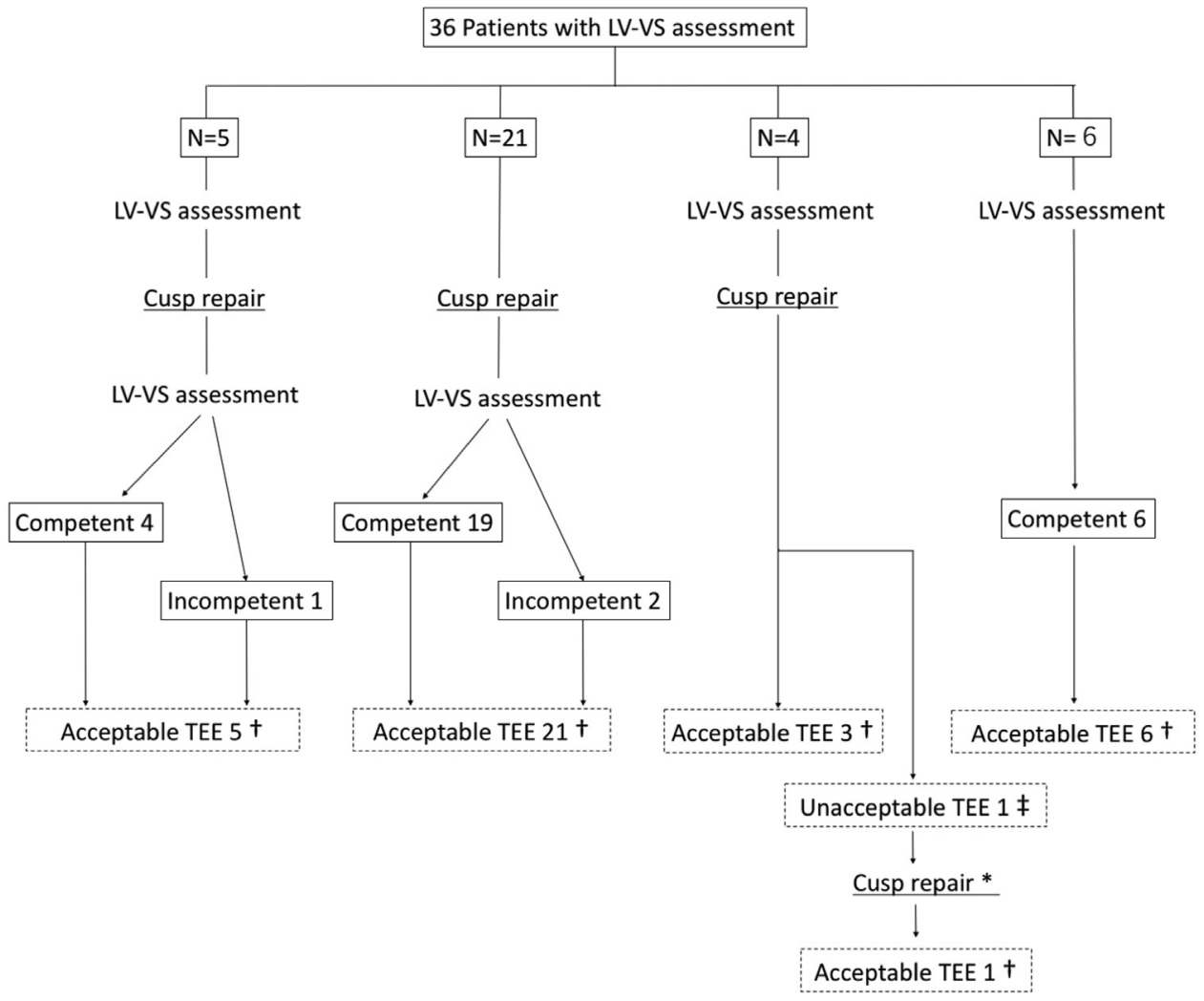

FIGURE 2. Flowchart of intraoperative management with LV-VS. *Revised cusp repair was performed both on mitral and aortic valve with secondary aortic cross-clamp. †Acceptable TEE means AR less than moderate. $\ddagger$ Unacceptable TEE means AR greater than mild to moderate. $L V$ - VS, Left ventricular videoscope; TEE, transesophageal echocardiography. 
TABLE 2. Intraoperative management of LV-VS and findings of LV-VS and TEE

\begin{tabular}{lccccccc}
\hline & & \multicolumn{2}{c}{ Postprocedural LV-VS findings } & & \multicolumn{3}{c}{ Postoperative TEE findings } \\
\cline { 3 - 4 } \cline { 6 - 8 } Group & No. patients* & Competent valve & Incompetent valve & & Non/trivial AR & Mild AR & Mild-to-moderate AR \\
\hline 1 & 23 & 20 & 3 & 15 & 9 & 3 & 0 \\
2 & 9 & 9 & 0 & & 9 & 0 \\
\hline
\end{tabular}

LV-VS, Left ventricular videoscope; TEE, transesophageal echocardiography; $A R$, aortic regurgitation. *Four patients without postprocedural LV-VS assessment were excluded.

eccentric jet disappeared after central plication on right coronary cusp; the LV-VS findings of this patient were, accordingly, a competent valve. This case was diagnosed finally with the use of TEE as trivial AR (Figure $3, C$ ). Postprocedural LV-VS in Figure 3, E, shows a significant leakage with wide and massive current, which was diagnosed as incompetent valve in LV-VS and finally as mild-to-moderate AR on TEE (Figure 3,E). Six patients in group 1 underwent only LV-VS assessment without concomitant cusp repair, and all 6 patients had a competent valve and no significant AR on TTE. Figure $3, F$, shows a competent valve after root reimplantation without cusp repair. In group 2, 9 patients were diagnosed as having a competent valve during postprocedural LV-VS and finally were diagnosed as non/trivial

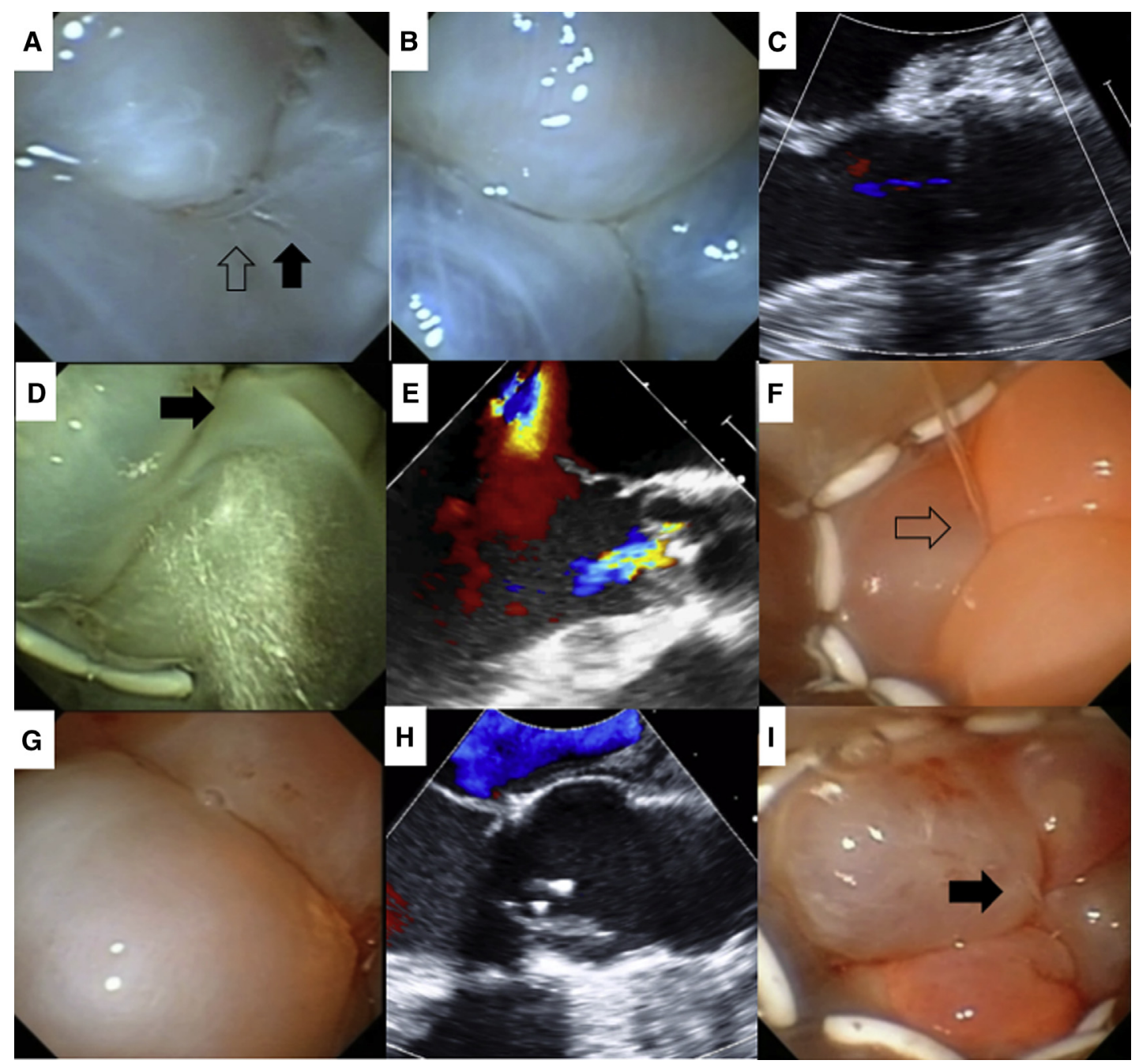

FIGURE 3. Pictures of LV-VS and corresponding TEE view. A, Preprocedural LV-VS in patients in group 1. Black arrow shows an eccentric jet due to prolapse of the right coronary cusp. Open arrow shows coexisting central jet. B, Postprocedural LV-VS shows competent valve with no AR. C, Postoperative TEE shows trivial AR without eccentricity. D, Postprocedural LV-VS shows wide and massive AR jet (black arrow) in patients in group 1. E, Postoperative TEE shows mild-to-moderate AR without eccentricity. F, A competent valve with narrow central ray (open arrow) in patient without cusp repair. G, Postprocedural LV-VS shows competent valve with bicuspid pathology. H, Postoperative TEE shows no AR. I, Quadricuspid aortic valve with residual eccentric jet (black arrow), which was managed with an additional cusp repair. 
AR in TEE. Figure 3, $G$, shows a competent valve with no leakage in patient with bicuspid aortic valve after free margin plication on a fused cusp. ${ }^{7}$ Postoperative TEE also showed no residual AR (Figure 3, H). Figure 3, I, shows a significant leakage of an eccentric jet from the bileafleted noncoronary cusp in patient with a quadricuspid aortic valve. An additional plication stitch was added at the edge of the noncoronary cusp for tricuspitalization, ${ }^{10}$ and the eccentric jet disappeared on TEE (Video 1).

\section{DISCUSSION}

During assessment after mitral valve repair, the closure line of the valve and absence of leakage usually are demonstrable on saline testing. ${ }^{11}$ Because we can observe the valve from the inflow side, the method of saline testing makes sense. An adequate aortic valve repair also should result in a competent valve with adequate surface of coaptation and the absence of major leakage. The method for assessing AR after aortic valve repair during cardiac arrest has not been established, however, and has been a matter of concern. During an operation, we have not able to see the unknown world below the aortic valve. Our LV-VS system provides clarification of the quality of the assessment after cusp repair by confirming an optimal closure of the valve with the direct view below the aortic valve. The procedure

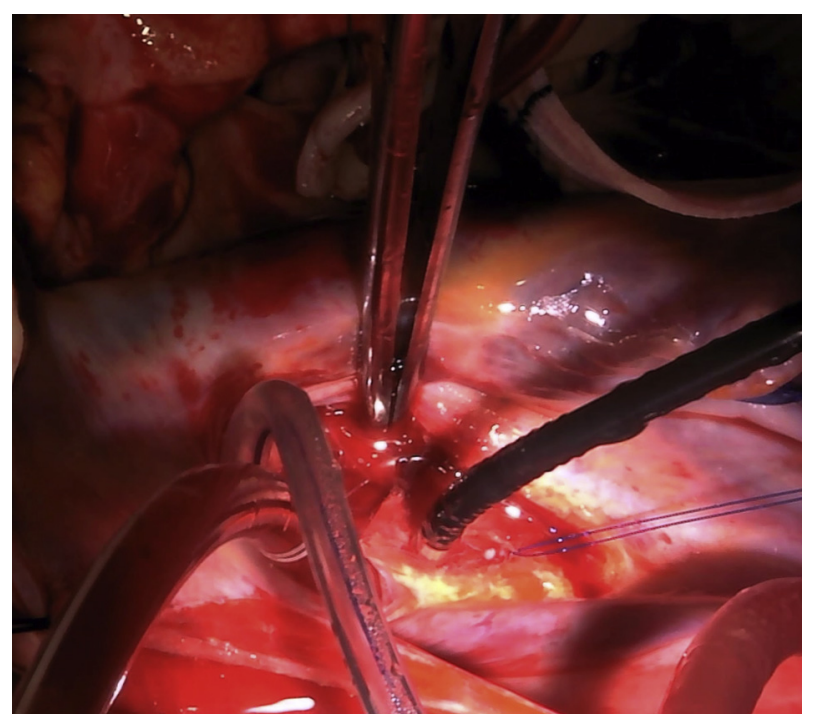

VIDEO 1. The LV-VS was inserted from the right upper pulmonary vein. When the scope reaches into the LV, we could see the tip of the scope through aortic valve and also the faces of the surgeons through the scope. Therefore, the surgeon can address the orientation of each cusp on the video image easily. The first and second cases in the video correspond with Figure 3, D, and Figure 3, F, in the manuscript, respectively. The surgeon also can manipulate the tip of the scope during setup through the aortic valve. In this regard, a forceps covered with rubber in its tip should be used, because the tip of the scope is very fragile and its coating can be peeled off easily. Video available at: http://www.jtcvsonline.org/article/ S0022-5223(17)30193-9/addons. to insert the scope could be performed in the same manner as the insertion of the LV vent; however, it took sometimes considerable time to set the position. This means a demand for longer crossclamp time, although it was at maximum 10 minutes. In addition, when a massive leak from aortic valve flooded the LV, we could not see anything through the scope.

In aortic valve-preserving surgery, type II regurgitant pathology is common, in which residual leakage, even if only slightly, will likely predispose the patient to recurrent AR. ${ }^{12}$ The group of Schäfers ${ }^{13}$ introduced a scientific measurement of cusp morphology using a specialized caliper, which can measure the height between the aortoventricular junction and the tip of the cusp coaptation. This "effective height" concept is theoretically proper to correct the cusp prolapse with objectivity and simplified these kinds of operation with reproducibility ${ }^{14}$; however, it is still debatable whether the cusp configuration under cardiac arrest also is preserved under pressurized aortic root.

Another German group recently demonstrated a method with the videoscope.$^{15}$ They laid the scope above the aortic valve, just as Itoh and colleagues ${ }^{16,17}$ previously reported. By this method, surgeon can detect the condition of the leaflet coaptation in the pressurized aortic root but not obtain detailed information about the residual leak: direction, orientation, and volume of the leakage. Our LVVS therefore might provide useful information regarding the mechanism and nature of the residual leakage by visualizing it directly.

Furthermore, the LV-VS enables us to immediately optimize the repair with adequate reinterventions before TEE, which requires resuscitation of the heart. In our experience, however, we have had 3 cases with incompetent valve in LV-VS assessment. Surgeons in these situations must decide whether or not to excise the valve for replacement. Our cases were accordingly diagnosed, at the most, as mild to moderate AR on TEE. The assessment of LV-VS might sometimes overestimate the degree of AR because of too-high aortic root pressure. Even if an artificially induced leak takes place in high root pressure, however, these kinds of leaks might not be significant, because promptly increasing root pressure also is one of the signs that confirm valve competency. It also would be helpful to compare the findings of LV-VS with the change over time of the root pressure, intend to grade the amount of residual AR. Furthermore, greater root pressure would able to clarify the eccentricity of the jet, in case it exists. In the present study, the small number of the patients with incompetent valves also prevented us from making a quantitative assessment of regurgitant jet with LV-VS.

We also applied LV-VS in patients who had no cusp pathology and no preoperative plan for concomitant cusp repair, because a certain number of patients treated with reimplantation technique has procedure-induced 
cusp prolapse. ${ }^{18}$ Our method could detect such subtle differences by observing the direct image below the aortic valve.

\section{CONCLUSION}

The findings of intraoperative assessment with LV-VS were consistent with the findings of postoperative TEE. We believe our strategy of using LV-VS has the potential to facilitate greater quality of valve assessment during valve-sparing root replacement with and without cusp repair. Further study is necessary to further correlate the LV-VS approach with echocardiographic and clinical outcomes.

\section{References}

1. Bailey CP, Zimmerman J. The surgical correction of aortic regurgitation; bicuspid conversion. Am J Cardiol. 1959;3:6-21.

2. Committee for Scientific Affairs, The Japanese Association for Thoracic Surgery, Masuda M, Okumura M, Doki Y, Endo S, Hirata Y, Kobayashi J, et al, Thoracic and cardiovascular surgery in Japan during 2014: annual report by The Japanese Association for Thoracic Surgery. Gen Thorac Cardiovasc Surg. 2016;64:665-97.

3. David TE, Feindel CM, David CM, Manlhiot C. A quarter of a century of experience with aortic valve-sparing operations. J Thorac Cardiovasc Surg. 2014; 148:872-9; discussion 879-80.

4. Miyahara S, Matsueda T, Izawa N, Yamanaka K, Sakamoto T, Nomura Y, et al. Mid-term results of valve-sparing aortic root replacement in patients with expanded indications. Ann Thorac Surg. 2015;100:845-51; discussion 852.

5. Langer F, Graeter T, Nikoloudakis N, Aicher D, Wendler O, Schafers HJ. Valvepreserving aortic replacement: does the additional repair of leaflet prolapse adversely affect the results? J Thorac Cardiovasc Surg. 2001;122:270-7.

6. Okita Y, Oka T, Miyahara S, Okada K. Direct visualization of the aortic cusp from the left ventricle during aortic root reimplantation. J Thorac Cardiovasc Surg. 2012;144:981-2.

7. Miyahara S, Abe N, Matsueda T, Izawa N, Yamazato T, Nomura Y, et al. Impact of positional relationship of commissures on cusp function after valve-sparing root replacement for regurgitant bicuspid aortic valve. Eur J Cardiothorac Surg. 2016;50:75-81

8. Matsumori M, Tanaka H, Kawanishi Y, Onishi T, Nakagiri K, Yamashita T, et al. Comparison of distensibility of the aortic root and cusp motion after aortic roo replacement with two reimplantation techniques: Valsalva graft versus tube graft. Interact Cardiovasc Thorac Surg. 2007:6:177-81.

9. Zoghbi WA, Enriquez-Sarano M, Foster E, Grayburn PA, Kraft CD, Levine RA, et al. Recommendations for evaluation of the severity of native valvular regurgitation with two-dimensional and Doppler echocardiography. J Am Soc Echocar diogr. 2003; 16:777-802.

10. Yamanaka K, Okada K, Okita Y. Aortic root replacement with a valve-sparing technique for quadricuspid aortic valve. Eur J Cardiothorac Surg. 2015;47:741-3

11. Carpentier A. Cardiac valve surgery - the "French correction." J Thorac Cardiovasc Surg. 1983;86(3):323-37.

12. Miyahara S, Omura A, Sakamoto T, Nomura Y, Inoue T, Minami H, et al. Impact of postoperative cusp configuration on midterm durability after aortic root reimplantation. J Heart Valve Dis. 2013;22:509-16.

13. Schäfers HJ, Bierbach B, Aicher D. A new approach to the assessment of aortic cusp geometry. J Thorac Cardiovasc Surg. 2006;132:436-8.

14. Kunihara T, Aicher D, Rodionycheva S, Groesdonk HV, Langer F, Sata F, et al Preoperative aortic root geometry and postoperative cusp configuration primarily determine long-term outcome after valve-preserving aortic root repair. J Thorac Cardiovasc Surg. 2012;143:1389-95.

15. Tsagakis K, Benedik J, El Khoury G, Jakob H. Aortic valve repair: intraoperative evaluation of valve geometry by angioscopy. J Thorac Cardiovasc Surg. 2015; 149:1666-8.

16. Itoh T, Ohtsubo S, Furukawa K, Norita H. Aortic root endoscopy in valve-sparing operations. J Thorac Cardiovasc Surg. 1997;114:141-2.

17. Furukawa K, Ohtsubo S, Itoh T. Aortic root endoscopy for aortic valve-sparing operations. J Thorac Cardiovasc Surg. 2016;152:638-9.

18. Boodhwani M, de Kerchove L, Watremez C, Glineur D, Vanoverschelde JL, Noirhomme P, et al. Assessment and repair of aortic valve cusp prolapse: implications for valve-sparing procedures. J Thorac Cardiovasc Surg. 2011;141: 917-25.

Key Words: aortic root replacement, aortic cusp repair, intraoperative assessment, echocardiography, aortic regurgitation

Readers who found these articles interesting may also like to read the following papers found in recent and future issues of our sister publications, Seminars in Thoracic and Cardiovascular Surgery and Operative Techniques in Thoracic and Cardiovascular Surgery!

\section{Acquired: Aortic Valve: Transcatheter}

News and Views: TAVR vs SAVR: Rising Expectations and Changing Indications for Surgery in Response to PARTNER II. Cristiano Spadaccio. Semin Thoracic Surg 2016: In press.

Original Submission: The Effect of Initiation of a TAVR Program on the Treatment of Severe Aortic Stenosis. Niv Ad. Semin Thoracic Surg 2016:353-360.

Editorial Commentary: Implementing the TAVR heart team: Complementary, Not Competitive, to SAVR volume And Outcomes. Xiaoying Lou. Semin Thoracic Surg 2016:361-362.

Original Submission: Transapical Cannulation Through a Transcatheter Aortic Valve Implantation Valve: A Novel Approach for Cardiogenic Collapse. Louis Philippe Tremblay. Semin Thoracic Surg 2016:400-402.

Editorial Commentary: Preparation for Transapical Arterial Access and Perfusion is Essential for Transcatheter Aortic Valve Replacement. Richard J. Shemin. Semin Thoracic Surg 2016:403. 